\title{
Visual function and course of basal laminar drusen combined with vitelliform macular detachment
}

\author{
S Y Cohen, I Meunier, G Soubrane, A Glacet-Bernard, G J Coscas
}

\begin{abstract}
Basal laminar drusen (BLD) are small round yellow drusen that are more easily visualised angiographically than biomicroscopically, with a 'stars in the sky' pattern. Patients with BLD are predisposed to macular vitelliform detachment. Little is known about the course of the disease, but the prognosis for retention of useful central vision for patients with BLD is thought to be better than for patients with typical drusen. A retrospective analysis of clinical and angiographic charts of 19 patients with BLD combined with a vitelliform macular detachment was performed to precisely describe their course. In addition, nine patients were re-examined to allow an analysis of their visual function - that is, central visual field, contrast sensitivity, and colour vision. Eyes without choroidal new vessels retained a fair visual acuity (mean final visual acuity 0.5 ; follow up 4 to 69 months, mean 24 months). In 11 of these eyes visual function assessment disclosed a reduction of contrast sensitivity in high and medium spatial frequencies in nine eyes ( $81 \%)$, a blue-yellow dyschromatopsia in nine eyes $(81 \%)$, and a mild reduction of foveal threshold in seven eyes (63\%). Choroidal neovascularisation (CNV) was observed in 12 eyes $(31 \%)$ with a poor final outcome (mean final visual acuity $0 \cdot 1$ ). Two thirds of cases of CNV were observed at the time of presentation; thus this finding may be a bias of a referring centre. However, the high prevalence of CNV suggests the need for a close follow up of patients with BLD.

(Br F Ophthalmol 1994; 78: 437-440)
\end{abstract}

Basal laminar drusen, or cuticular drusen, were first described in 1985 by Gass et al. ${ }^{1}$ Histological examination disclosed a different pattern from typical drusen - that is, a nodular thickening of the basement membrane of the retinal pigment epithelium (RPE). Basal laminar drusen should not be confused with basal linear deposit which is a histological finding observed in the early stages of age-related macular degeneration. ${ }^{2}$

Basal laminar drusen (BLD) are small round yellow drusen that are more easily visualised angiographically than biomicroscopically, with a 'stars in the sky' or a 'milky way' pattern. Basal laminar drusen present a fluorescence which is more intense in the early arteriovenous phase of the sequence (Fig 1). They are usually observed in the fourth or the fifth decade. They may be associated with typical drusen, or more often with a vitelliform macular detachment. It should be noted that the association of drusen and adult vitelliform macular lesions was reported by Marmor in 1979 in one case of a study about vitelliform lesions in adults, and this case is probably the first published one of BLD combined with vitelliform macular detachment. ${ }^{3}$ In the initial description of the disease by Gass et al, the material spontaneously resolved in $50 \%$ of cases. No case of choroidal new vessels was observed during the follow up. Visual prognosis in patients with BLD was estimated to be better than in patients with typical drusen.'

To our knowledge, since this initial report, no study was performed on the spontaneous evolution of BLD combined with vitelliform macular detachment. We performed a retrospective analysis of clinical and angiographic charts of 19 patients with BLD combined with a vitelliform macular detachment. In addition, nine patients were re-examined to allow an analysis of their visual function - that is, central visual field, contrast sensitivity, and colour vision.

Patients and methods

Clinical and angiographic charts of patients with BLD combined with vitelliform macular detachment examined from 1985 to 1991 in the Department of Ophthalmology of Creteil, France were reviewed. For each patient were noted: sex, age at presentation, initial and final visual acuity, results of fundus examination and of fluorescein angiography. All the patients underwent fluorescein angiography at the time of presentation and at follow up. All patients were asked to return for a new examination. The patients who were re-examined had a new fluorescein angiography, and before pupillary dilatation, an automated perimetry (Humphrey field analyser, Zeiss), a contrast sensitivity function assessment

Figure 1 Basal laminar drusen and vitelliform macular detachment in a 46-year-old woman. The arteriovenous phase of the angiogram showed a 'milky way' pattern of the fundus owing to the widespread hyperfuorescence of the drusen. The staining of the central detachment appears inhomogeneous. 


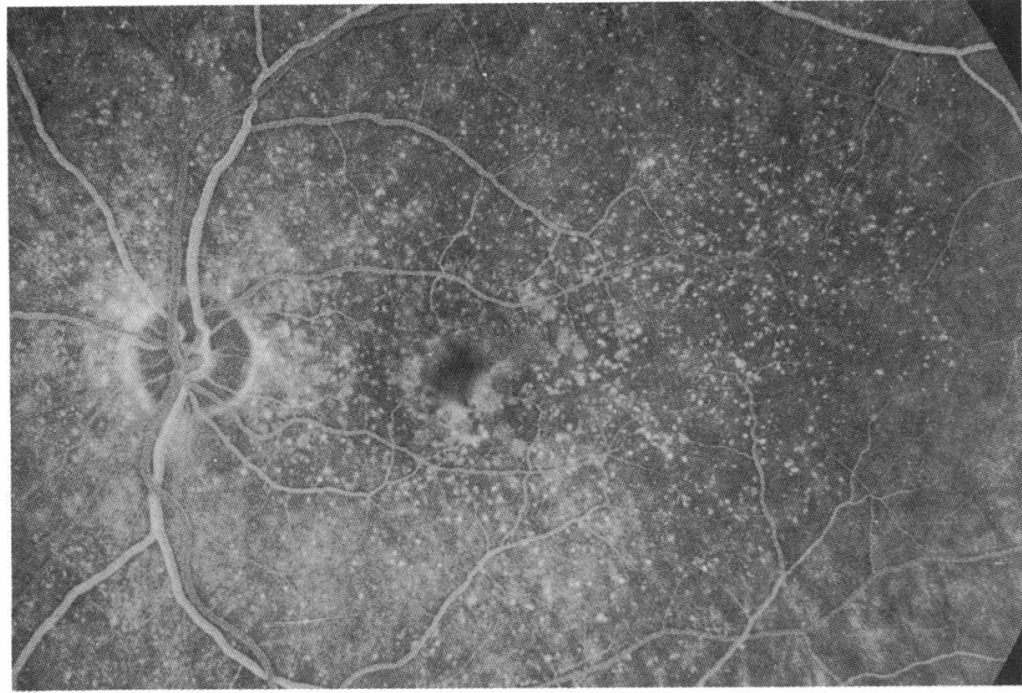

Fig $2 A$

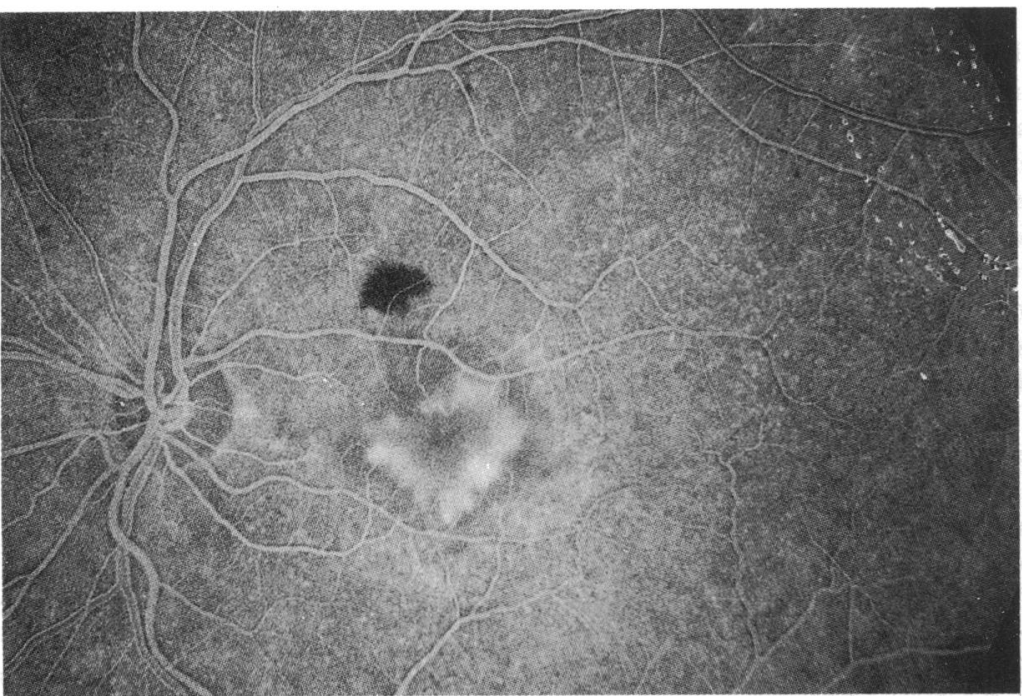

Fig $2 B$

Figure 2 (A) Basal laminar drusen and vitelliform macular detachment in a 75-year-old man. The arteriovenous phase of the angiogram showed a hyperfluorescence of the drusen and of the vitelliform detachment (B) Same patient, 3 years later. Appearance of a subfoveal choroidal neovascularisation. contrast needed was compared with the level of normal age-matched subjects. A ratio of 4 between the two levels (that is, level of contrast needed in patient/level of contrast needed in normals) was considered as significant for medium and high spatial frequencies. When patients could not see the bar grating corresponding to $20 \mathrm{cpd}$, they were noted as 'out of scale' and the ratio was considered significant.

\section{Results}

From 1985 to 1991,19 patients with BLD combined with vitelliform macular detachment were examined. There were 13 women $(68.5 \%)$ and six men. They were aged 44 to 77 years (mean 61.4) years. The follow up was 4 to 69 months (mean 24 months). Nine patients were re-examined, and visual function examination was performed in 11 eyes.

At presentation, we asked patients about familial ocular disease. We had no positive response. Initial visual acuity varied from 0.01 to 1 , with a mean visual acuity of $0 \cdot 47$. A visual acuity of 0.5 or better was found in $50 \%$ of the eyes. Basal laminar drusen were always bilateral. A vitelliform material was present in each case, and was always bilateral. The material was multiple in one eye, and presented a pseudohypopyon pattern in five eyes. Mild to moderate pigmentary migrations were observed in 28 eyes of 16 patients. Typical drusen were associated with the BLD in 24 eyes of 12 patients; these drusen were hard in nine cases, soft in nine cases, and soft and hard in six other cases.

Choroidal neovascularisation (CNV) was observed, at presentation, in eight eyes of seven patients. During the follow up, two patients developed CNV in both eyes (Fig 2). At the end of the follow up, CNV had been observed in $31 \%$ of the eyes (12 of 38 eyes) and in $47 \%$ of patients (nine out of 19 patients). The neovascular membrane was well defined in six cases and ill defined in six other cases. Six eyes were treated with krypton laser; four recurrences were observed. In eyes with $C N V$, final visual acuity varied from 0.005 to 0.3 with a mean final visual acuity of $0 \cdot 1$.

In eyes without choroidal neovascularisation, initial visual acuity varied from 0.2 to 1 , with a mean initial visual acuity of $0 \cdot 57$. Final visual acuity varied from 0.2 to 0.8 , with a mean final visual acuity of $0 \cdot 5$. These eyes retained a fair visual acuity but presented moderate disturbances of visual function. We observed the following results of the visual function assessment (11 eyes of nine patients): a mild reduction of foveal threshold in seven eyes (63\%) (Table 1); a reduction of contrast sensitivity in high and medium spatial frequencies in nine eyes $(81 \%)$ (Table 1); and a blue-yellow dyschromatopsia in nine eyes (81\%) (Fig 3). No case of spontaneous resolution of the material was observed. A fragmentation of this material occurred in three cases, and the appearance of small areas of atrophy of the RPE was observed in one case; however, we did not see any case of complete resolution of the material with constitution of a large area of geographic atrophy.

The global visual outcome was as follows: mean final visual acuity was 0.38 (mean initial spatial frequencies with bar gratings corresponding to $20 \mathrm{cpd}$. For these bar gratings, the level of 
Table 1 Results of visual function assessment in nine patients

\begin{tabular}{llll}
\hline \multirow{2}{*}{$\begin{array}{l}\text { Patients } \\
\text { (eyes) }\end{array}$} & $\begin{array}{l}\text { Reduction of } \\
\text { foveal threshold } \\
(d B)\end{array}$ & \multicolumn{2}{c}{ Ratio of contrast levels } \\
\cline { 3 - 4 } \cline { 3 - 4 } l(RE) & 6 & $3 c p d$ & $20 c p d$ \\
\hline (RE) & 6 & 10 & $5 \cdot 3$ \\
3(RE) & 7 & 6 & $5 \cdot 3$ \\
(LE) & 11 & 6 & 8 \\
4(RE) & 4 & 10 & Out of scale \\
5(LE) & 3 & 1 & Out of scale \\
6(RE) & 4 & 4 & $5 \cdot 3$ \\
(LE) & 3 & 1 & $1 \cdot 6$ \\
7(RE) & 5 & 6 & Out of scale \\
8(LE) & 10 & 6 & Out of scale \\
9(RE) & 12 & 6 & Out of scale \\
\hline
\end{tabular}

visual acuity 0.47$) ; 16$ out of 38 eyes (42\%) retained a visual acuity of 0.5 or better; 12 patients out of $19(63 \%)$ retained a visual acuity of 0.5 or better in at least one eye.

\section{Discussion}

Basal laminar drusen were described by Gass et al in $1985 .{ }^{1}$ In this preliminary publication, the authors distinguished these peculiar drusen from typical drusen by clinical and histological findings. Electron microscopic examinations suggested that basal laminar drusen were caused by focal nodular thickening of the pigment epithelium basement membrane ${ }^{14}$ whereas typical drusen are caused by focal detachment of the pigment epithelium and its normal thickness basement membrane from the remainder of Bruch's membrane. ${ }^{5}$ If there is clinical and histological evidence to support the concept of nodular thickening of the basement membrane of the retinal pigment epithelium as the cause for basal laminar drusen, the nature of the yellow macular subretinal exudate is still unknown. This yellow vitelliform macular detachment may stimulate the lesions seen in Best's disease or in adult vitelliform macular dystrophy. Best's disease is known to be inherited with an autosomal dominant transmission, and adult vitelliform macular dystrophy is thought to be transmitted in the same way. ${ }^{6}$ To our knowledge, a familial involvement has never been reported in

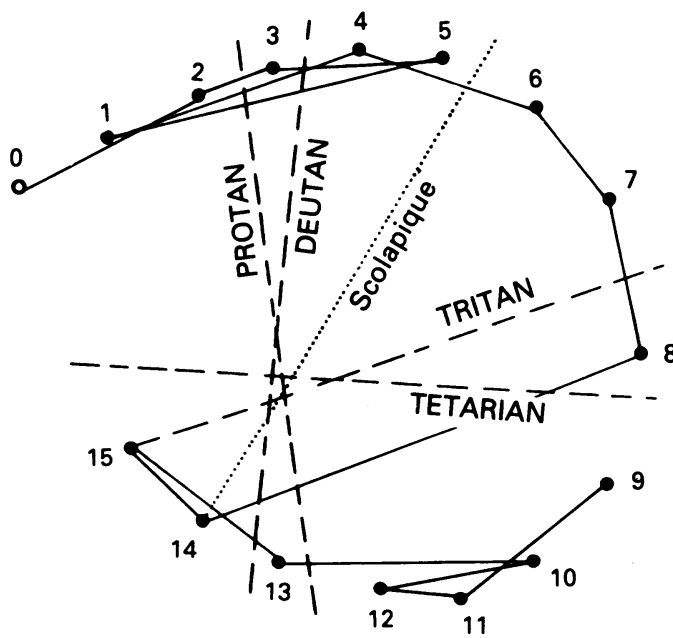

Figure 3 Typical blue-yellow dyschromatopsia observed with the Lanthony desaturated Farnsworth 15 hue in a

54-year-old woman with basal laminar drusen combined with a vitelliform macular detachment. basal laminar drusen combined with a vitelliform macular detachment.

Basal laminar drusen seemed to predispose patients, particularly in later life, to development of larger typical drusen in the macula and a yellow exudative retinal detachment. In Gass's series, 17 of 41 patients developed visual loss caused by vitelliform macular detachment of one or both eyes ( 26 eyes). The mean age was 63 years in these 17 patients, ${ }^{1}$ similar to our mean value (61.5 years). BLD combined with vitelliform macular detachment occur more commonly in women in both series, with almost the same ratio of two women for one man. Most of the eyes with vitelliform macular detachment and without choroidal neovascularisation had visual acuity of 0.3 or better in each study.

According to Gass et al, spontaneous resolution of the macular detachment and retention of good visual acuity occurred frequently. ${ }^{1}$ Thus, macular detachment observed in 18 eyes has resolved in 10 eyes for a mean 57 months of follow up (range, 24 to 97 months). After resolution, the visual acuity was 0.5 or better in nine of the 10 eyes. The area of persistent macular detachment (eight eyes) was enlarged in four eyes and unchanged in four eyes. Our findings were not similar. None of the 38 eyes presented evidence of spontaneous resolution of the detachment, but the follow up was less (mean follow up 24 months). In all eyes but one, the area of macular detachment was unchanged or enlarged.

Choroidal neovascularisation is the main complication of BLD. In the study by Gass et al, only four patients presented with choroidal neovascularisation and no case appeared during the follow up. In the present study, choroidal neovascularisation was observed in 12 out of 38 eyes $(31 \%)$. Two thirds of the cases of CNV were observed at the time of presentation; thus, there may be a bias of referring patients when $\mathrm{CNV}$ occurred. These eyes presented a poor visual outcome.

In the present study, central automated perimetry disclosed a central depression with a mild reduction of foveal threshold in $63 \%$ of cases. Visual function disorders have been previously described in adult vitelliform macular dystrophy ${ }^{7}$ with such a reduction of foveal threshold. The reduction of contrast sensitivity in high and medium frequencies had no specificity and could be observed in other maculopathies. However, such a reduction was observed in the present study with a very high prevalence $(81 \%$ of cases). Colour vision testing results support evidence of a blue-yellow dyschromatopsia in $81 \%$ of eyes with BLD and vitelliform macular detachment. Patients with adult vitelliform macular dystrophy usually showed normal colour vision or non-specific colour vision defect. ${ }^{7}$ The use of the Lanthony desaturated Farnsworth 15 hue may have helped to diagnose mild dysfunctions of the colour vision, because the test is known for its high sensibility in detecting the acquired dyschromatopsia. ${ }^{8}$

In conclusion, patients with basal laminar drusen combined with vitelliform macular detachment presented moderate disturbances of visual function. Choroidal neovascularisation is 
the main complication of the disease, observed in the present study in $31 \%$ of the eyes. This prevalence of choroidal neovascularisation could be partially overestimated by a referring bias, as two thirds of CNV were observed at the time of presentation. However, it suggests the need for a close follow up of patients with BLD.

Presented as a poster at the American Academy of Ophthalmology Annual Meeting, Dallas, November 1992.

1 Gass JDM, Jallow S, Davis B. Adult vitelliform macular detachment occurring in patients with basal laminar drusen. Am F Ophthalmol 1985; 99: 445-59.
2 Sarks SH. Ageing and degeneration in the macular region. A clinico-pathological study. Br f Ophthalmol 1976; 60 $324-41$.

3 Marmor MF. 'Vitelliform' lesions in adults. Ann Ophthalmol 1979; 11: 1705-12.

4 Kenyon KR, Maumenee AC, Ryan SJ, Whitmore PV, Green WR. Diffuse drusen and associate complications. Am Ophthalmol 1985; 100: 119-28.

5 Green WR, Key SN. Senile macular degeneration: a histopathological study. Trans Am Ophthalmol Soc 1977; 75: 180-254.

6 Brecher R, Bird AC. Adult vitelliform macular dystrophy. Eye

7 Sabates R, Pruett RC, Hirose T. Pseudovitelliform macular degeneration. Retina 1982; 2: 197-205.
d

8 Lanthony $P$, Dubois-Poulsen A. Le Farnsworth 15 desaturé. Bull Soc Ophtalmol Fr 1973; 73: 861-6. 